\title{
Relationship Between Estimated Glycosylated Hemoglobin Using Flash Glucose Monitoring and Actual Measured Glycosylated Hemoglobin in a Chinese Population
}

Yun Hu · Yun Shen · Rengna Yan · Fengfei Li · Bo Ding •

Huiying Wang · Xiaofei Su · Jianhua Ma (D)

Received: January 12, 2020 / Published online: July 21, 2020

(C) The Author(s) 2020

\section{ABSTRACT}

Introduction: The aim of this study was to investigate the relationship between actual measured glycated hemoglobin (HbA1c) and estimated glycated hemoglobin (EA1c) in the flash glucose monitoring (FGM) system in Chinese patients with type 2 diabetes.

Methods: This study was conducted in Nanjing First Hospital. Each patient used FGM twice in a 3-month period (during the first 14 days immediately after baseline and during a second 14-day period from days 76 to 90 after baseline). HbA1c measurements were made using a highperformance liquid chromatography assay before the start of the first FGM period (baseline) and at the end of the second FGM period. Results: A total of 74 patients (35 men; mean age \pm standard deviation [SD] $67.6 \pm 5.2$ years)

Yun $\mathrm{Hu}$, Yun Shen, Rengna Yan contributed equally to this article.

Digital Features To view digital features for this article go to https://doi.org/10.6084/m9.figshare.12600074.

Y. Hu · Y. Shen · R. Yan · F. Li - B. Ding · H. Wang ·

X. Su $(\bowtie) \cdot J . M a(\bowtie)$

Department of Endocrinology, Nanjing First

Hospital, Nanjing Medical University, Nanjing,

Jiangsu, China

e-mail: suxiaofeifei@126.com

J. Ma

e-mail: majianhua196503@126.com were enrolled in the study. The mean ( \pm SD) duration of diabetes was $11.9 \pm 7.8$ months. The first and second HbA1c measurements were both higher than the EA1c (both $p<0.001$ ). Mean glucose (MG) gradually decreased over time and was the lowest on day 14. Linear regression showed that only $\mathrm{HbA1c}$ at baseline affected the gap between HbA1c and EA1c $(\beta=$ $0.319, p=0.01$ ) when the educational level, age, gender, duration of diabetes, body mass index, HbA1c at baseline, and number of scans daily were included as independent variables. The best model for calculating EA1c was EA1c\% = MG $\mathrm{mmol} / \mathrm{L} \times 0.669-0.213 \times 8$ th $\mathrm{MG}+$ 3.351 when $\mathrm{MG}>9.7 \mathrm{mmol} / \mathrm{L}$, and $\mathrm{EA} 1 \mathrm{c}$ $\%=(\mathrm{MG} \quad \mathrm{mmol} / \mathrm{L}+2.590) / 1.590$ when $\mathrm{MG} \leq 9.7 \mathrm{mmol} / \mathrm{L}$. The correlation coefficient for EA1c and HbA1c in this model (model 7) is higher than that reported the original model in the FGM system 1 (0.955 vs. 0.822 , respectively; $p<0.001)$.

Conclusions: The EA1c used by FreeStyle Libre $^{\mathrm{TM}}$ is lower than the actual measured HbA1c. Improvement in the glucose levels during FGM in these patients may contribute to the lowering of EA1c.

Trial Registration: The study is registered with ClinicalTrials.gov, number NCT03785301.

Keywords: Estimated glycated hemoglobin; Flash glucose monitoring; Glycated hemoglobin 


\section{Key Summary Points}

Flash glucose monitoring can improve blood glucose in the short term of 14 days without the need to adjust hypoglycemic treatment.

The estimated A1c used by FreeStyle Libre $^{\mathrm{TM}}$ is lower than actual glycated hemoglobin (HbA1c).

The gap between estimated A1c and actual measured $\mathrm{HbA1c}$ is positively correlated to the actual HbA1c.

Based on our results, we present an improved model for estimating A1c in flash glucose monitoring.

\section{INTRODUCTION}

The FreeStyle Libre ${ }^{\mathrm{TM}}$ (Abbott Diabetes Care, Witney, UK) flash glucose monitoring (FGM) system is one of a new generation of glucosetesting devices. The FGM system has made glucose checks easier with its ability to scan a sensor for a glucose reading at any time, no need for calibration, and a long sensor lifetime of 14 days. Many studies have confirmed the benefit of using the FGM in patients with type 1 or type 2 diabetes [1-4], including reductions in hyperglycemia, hypoglycemia, and associated medical costs.

Glycated hemoglobin (HbA1c) is considered to be the gold standard for the assessment of glycemic control [5] and has strong predictive value for diabetes complications [6] and mortality in patients with diabetes [7]. The relationship between HbA1c and average glucose levels has been explored in many studies, most of which used intermittent capillary blood glucose measurements $[8,9]$ or continuous glucose monitoring (CGM) $[10,11]$, resulting in a formula that allowed the calculation of an estimated A1c value using average glucose. The calculation, namely, estimated glycated

hemoglobin (EA1c), calculated as $\mathrm{A} 1 \mathrm{c} \%=($ mean glucose $[\mathrm{MG}] \mathrm{mmol} / \mathrm{L}+2.59) / 1.59$ using the glucose data recorded by an FGM sensor for 14 days, was installed by Abbott Diabetes Care as a parameter in the FGM system. In some studies [1,2] and clinical work, EA1c has been used to assess glycemic control, rather than the actual HbA1c. Dunn and colleagues analyzed the effects of FGM in more than 50,000 users worldwide and found that both EA1c and time in hyperglycemia were gradually reduced with increasing scan rate from the lowest to highest scan groups [2]. EA1c has also been used by clinicians to guide adjustments in the hypoglycemic regimen in a large number of patients. The use of EA1c instead of actual HbA1c measurements can be partially explained by the desire to reduce blood collections and hospital visits for HbA1c testing. However, an analysis of the relationship between EA1c and actual measured HbA1c has never been performed. Thus, the aim of the present study was to investigate the relationship between EA1c as measured in a FGM system and actual measured HbA1c in Chinese patients with type 2 diabetes.

\section{METHODS}

\section{Patients}

This study was conducted in Nanjing First Hospital from December 2018 to July 2019. Patients with type 2 diabetes considered eligible to be enrolled in the study were (1) aged $\geq$ 18 years, (2) had $\mathrm{HbA} 1 \mathrm{c}<11.0 \%$; and (3) were drug naïve or in stable hypoglycemic therapy for at least 3 months prior to the study. Exclusion criteria were: (1) pregnancy or currently attempting to conceive; (2) presence of infection or acute metabolic complications of diabetes, such as ketoacidosis or hyperosmolar state (coma); (3) allergy to medical grade adhesive or isopropyl alcohol used to prepare the skin; (4) presence of skin lesions, scarring, redness, infection, or edema at sensor application sites; (5) presence of anemia or other diseases that may affect HbA1c testing; (6) presence of any other condition considered to warrant 
exclusion according to the researchers involved in the study.

The study protocol was approved by the Institutional Ethics Committee of Nanjing First Hospital, Nanjing Medical University (number KY20170904-04), and was in accordance with the Helsinki Declaration of 1964, as revised in 2013. All patient provided written informed consent forms to participate in the study.

\section{Study Design}

Glycated hemoglobin was measured using a high-performance liquid chromatography assay (Bio-Rad Laboratories, Inc., Hercules, CA, USA) at baseline, and FGM was used for 14 days in patients after the baseline blood collection (period 1). The sensor was worn on the back of the upper arm to record subcutaneous interstitial glucose concentration at 15 -min intervals without the requirement for self-monitoring of blood glucose (SMBG) for calibration. Patients could scan the sensor to read the glucose level at any time. The frequency of scanning by each patient for each sensor was calculated by counting the number of scans divided by the duration of sensor use (14 days), according to recorded start and end times. Patients were required to keep track of their food intake and exercise while wearing the FGM sensor and could alter their diet and exercise according to the glucose levels provided by FGM. Patients were also instructed to maintain their therapy with glucose-lowering agents during FGM in order to prevent the effects of a large glycemic variation caused by drug changes on the EA1c in FGM. The dosage of glucose-lowering agents could be adjusted by clinicians according to the results of the FGM and HbA1c when the FGM sensors were removed. To diminish the influence of any changes in diet and exercise on the EA1c, readings from FGM were also taken from days 76 to $90( \pm 2)$ (period 2) and the HbA1c was measured again when at the end of this second FGM period. The height, weight, course of disease, concomitant diseases, and medication of all patients were recorded.

\section{Statistical Analysis}

All statistical analyses were performed using SPSS version 16.0 software (IBM Corp., Armonk, NY, USA). Data are presented as the mean \pm standard deviation (SD) or as percentages. Differences between the EA1c and $\mathrm{HbA} 1 \mathrm{c}$ were examined using Student's paired $t$ test and univariate tests. Differences in daily MG level during the 14-day study period were analyzed by repeated measures analysis of variance (ANOVA) followed with a Bonferroni test. The correlations of EA1c and HbA1c in the two FGM periods were compared using the cocor package (https://comparingcorrelations.org/). The models for the relationship between EA1c and HbA1c were analyzed using stepwise linear regression and exponential and quadratic equations. Total MG and the MG for each day, scanning frequency, and the SD of glucose levels in the FGM system were used to construct the models. The best fits, based on assessment of correlation coefficient values closest to 1.0 using Pearson correlations, were determined. Stepwise linear regression was also performed to test potential risk factors possibly influencing the gap between EA1c and HbA1c (HbA1c EA1c); these variables were included as independent variables. $p$ values $<0.05$ were considered to indicate statistical significance.

\section{RESULTS}

\section{Patient Characteristics}

A total of 80 patients were enrolled in the study, of whom six were excluded from the analyses due to interruptions in FGM. The final study population thus comprised 74 patients, of whom $35(47.3 \%)$ were men; the mean age $( \pm$ SD) of the patients was $67.6 \pm 5.2$ years. At baseline, the mean duration of type 2 diabetes was $11.9 \pm 7.8$ months, mean body mass index (BMI) was $25.1 \pm 3.8 \mathrm{~kg} / \mathrm{m}^{2}$, and mean HbA1c was $7.7 \pm 1.3 \%$. Fifty-two $(70.3 \%)$ patients were on insulin therapy, and two patients were drug naïve. The baseline characteristics of the patients are given in Table 1. 
Table 1 Baseline characteristics of patients

\begin{tabular}{ll}
\hline Patient characteristics & Values \\
\hline Age (year) & $67.6 \pm 5.2$ \\
Sex (male) & $35(47.3 \%)$ \\
Diabetes duration (months) & $11.9 \pm 7.8$ \\
BMI (kg/m $\left.{ }^{2}\right)$ & $25.1 \pm 3.8$ \\
HbAlc (\%) & $7.7 \pm 1.3$ \\
Systolic blood pressure (mmHg) & $139.7 \pm 16.7$ \\
Smoking & $14(18.9 \%)$ \\
Hypertension & $53(71.6 \%)$ \\
DKD & $6(8.1 \%)$ \\
Retinopathy & $5(6.8 \%)$ \\
Neuropathy & $13(17.6 \%)$ \\
Carotid plaque & $24(32.4 \%)$ \\
Family history of diabetes & $42(56.8 \%)$ \\
Insulin use & $52(70.3 \%)$ \\
Sulfonylurea use & $28(37.8 \%)$ \\
Metformin use & $30(40.5 \%)$ \\
Thlucosidase use & $35(47.3 \%)$ \\
DPP-4 inhibitor use & $4(5.4 \%)$ \\
\hline Glinide use & $3(4.05 \%)$ \\
\hline
\end{tabular}

Data are presented as the mean \pm standard deviation $(\mathrm{SD})$, or as a number with the percentage in parenthesis $B M I$ Body mass index; $D K D$ diabetic kidney disease; $D P P$ 4 dipeptidyl peptidase-4, HbAlc glycated hemoglobin

\section{Differences between EA1c and Actual Measured HbA1c}

The HbA1c at baseline was significantly higher than the EA1c $(7.7 \pm 1.3$ vs. $7.1 \pm 1.3 \%$, respectively; $p<0.001$ ). After 3 months (period $2)$, the HbA1c remained higher than the EA1c $(7.5 \pm 1.1 \quad$ vs. $\quad 6.9 \pm 1.1 \%$, respectively; $p<0.001)$, although it should be noted that the actual HbA1c measurement was made after the second period of FGM.

\section{Mean Daily Glucose During FGM}

The repeated ANOVA revealed that there was no difference in MG between the two periods of FGM $(8.8 \pm 2.2$ vs. $8.6 \pm 1.9 ; p=0.663)$. However, the daily MG reading changed with time ( $F=7.2, p<0.001$ in the multivariate tests). To assess the influence of these changes in blood glucose on the EA1c, we pooled the FGM data from the two FGM periods and calculated the MG of each day (see Fig. 1). The MG was highest on the third day and then gradually decreased; this reduction in MG compared to the third day became statistically significant on the tenth day ( $p=0.015$ ), and MG on the 14th day was the lowest readings. MG on the first day was slightly lower than that on the second and third days ( $p=0.070$ and 0.054 , respectively), and there was no difference between MG on the second and third days $(p=0.722)$.

\section{Factors Influencing Difference between EA1c and Actual Measured HbA1c}

To explore the factors that have the potential to influence the difference between EA1c and HbA1c $(\triangle \mathrm{A} 1 \mathrm{c}=\mathrm{HbA1c}-\mathrm{EAlc})$, we performed

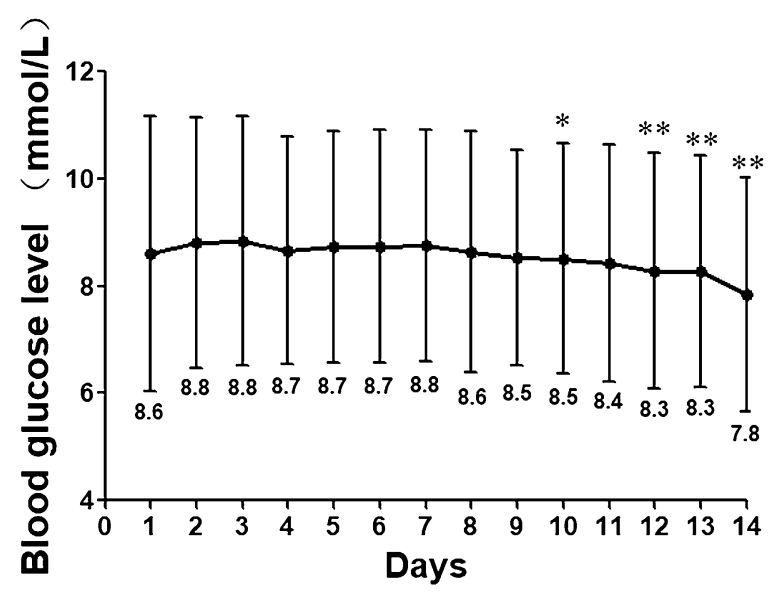

Fig. 1 Daily mean glucose of the patients during 14 days of flash glucose monitoring (FGM). Values are pooled data from two 14-day FGM monitoring periods. Asterisk(s) indicates a signficance difference in mean glucose (MG) as compared to MG on third day of 14-day FGM $\left({ }^{*} p<0.05 ;{ }^{* *} p<0.01\right)$ 
linear regression, including educational level, age, gender, duration of diabetes, BMI, HbA1c at baseline and times of daily scan as independent variables in the model. The results showed that only $\mathrm{HbA} 1 \mathrm{c}$ at baseline affected the $\Delta \mathrm{A} 1 \mathrm{c}$ $(\beta=0.319, p=0.010)$. We then combined the baseline and endpoint data and observed that $\Delta \mathrm{A} 1 \mathrm{c}$ increased with increasing HbA1c $(r=0.316, p<0.001)$, as shown in Fig. 2. The frequency of scanning [number of scans daily $=11.9$ (interquartile range 7.7-15.9)] was correlated to EA1c and HbA1c $(r=-0.164$ and $-0.225, p=0.016$ and 0.006 , respectively). However, there was no correlation between scan rates and $\Delta \mathrm{A} 1 \mathrm{c}(p>0.05)$.

\section{Correlation Analysis between EA1c and Actual Measured HbA1c}

Pearson analysis was performed to investigate the relationship between EA1c and the actual measured HbA1c, with both EA1c values found to be significantly and positively correlated to the real HbA1c values $(r=0.752$ and 0.810 , respectively; both $p<0.001)$. The correlations between EA1c and the actual measured HbA1c in the first and second FGM periods were similar $(p=0.255)$. Thus, we combined the EA1c from these two FGM periods and the values from the

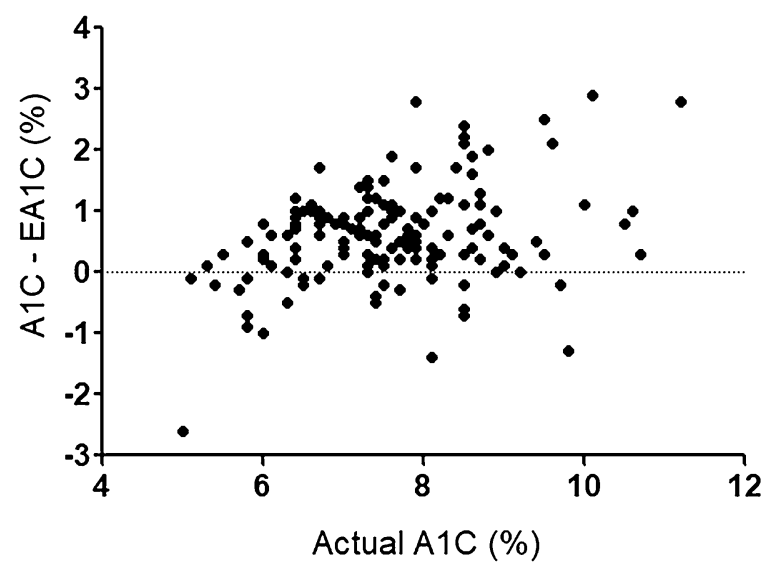

Fig. 2 Relationship between glycated hemoglobin (HbAlc) and the gap between actual measured HbAlc (Alc) and estimated Alc (EAlc) during FGM. A total of $148 \mathrm{HbAlc}$ measurements and the corresponding EAlc in the FGM system were included in linear regression two HbA1c tests; the correlation coefficient was $0.822[p<0.001(n=148)]$. We then performed linear regression under different conditions to search for a better model of EA1c (shown in Table 2). Models 1 to 4 in Table 2 were calculated using MG only. The correlation coefficient in models 2 to 4 was not higher than the previous EA1c and was lower in the exponential and quadratic equation than in the linear regression. Given the influence of scanning frequency and variation in daily glucose levels, in the linear regression for models 5 to 8 we included MG on the second to 14th day of FGM, the SD of glucose levels (which represented glycemic variability), and the number of scans daily. In addition, given the strong effect of baseline HbA1c, which indicated blood glucose control, on $\Delta \mathrm{A} 1 \mathrm{c}$, in two models we stratified the patients by $M G$ (median value in model 6; by quartile in model 7). The best model was found to be $\mathrm{EA} 1 \mathrm{c} \%=\mathrm{MG} \mathrm{mmol} / \mathrm{L} \times$ $0.669-0.213 \times 8$ th $\mathrm{MG}+3.351$ when $\mathrm{MG}>$ $9.7 \mathrm{mmol} / \mathrm{L}$ and $\mathrm{EA} 1 \mathrm{c} \%=(\mathrm{MG} \mathrm{mmol} / \mathrm{L}+$ 2.590)/1.590 when $\mathrm{MG} \leq 9.7 \mathrm{mmol} / \mathrm{L}$ (model 7 in Table 2). The correlation coefficient in model 7 was significantly higher than that in model 1 (previous EA1c) $(p<0.001)$.

\section{DISCUSSION}

The results of our study reveal that in our patient population there was a gap between the EA1c in the FreeStyle Libre FGM system and the actual measured $\mathrm{HbA1c}$ and that this gap between EA1c and actual HbA1c was positively correlated to the levels of baseline HbA1c. Therefore, we conclude that EA1c should be calculated with the MG and adjusted using MG on the eighth day of FGM when MG is at a high level because the FreeStyle Libre FGM system itself has the effect reducing blood glucose (Table 2).

The difference between EA1c and actual measured HbA1c is mainly caused by the reduction in MG. Several studies have reported the effects of FGM on blood glucose [12, 13]. However, these studies were conducted in patients who were taking insulin therapy, and the insulin doses were adjusted during FGM. 


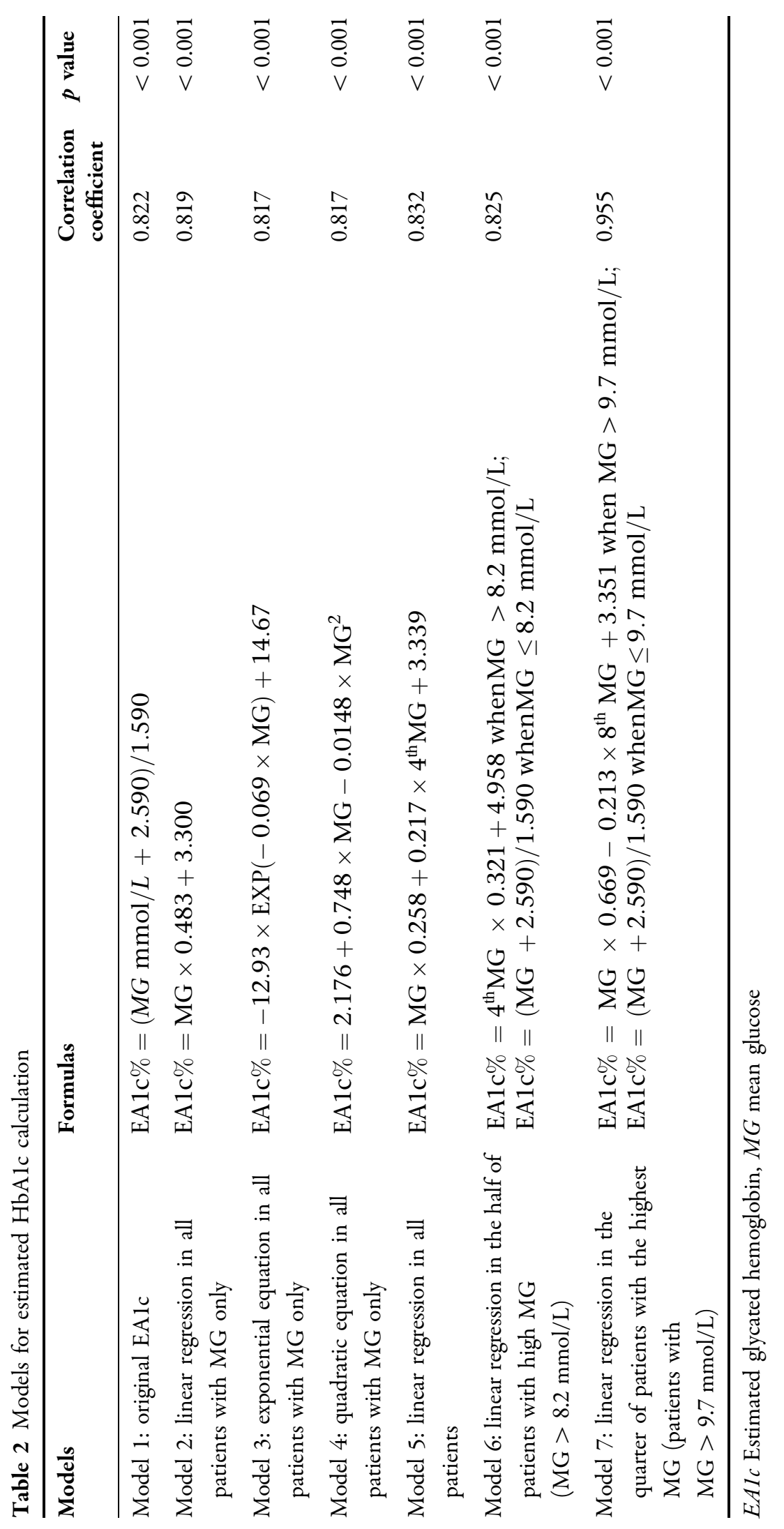


Moreover, these studies had durations of $>$ 10 weeks. We found that the MG decreased significantly in a short period of 14 days without any change in the use of glucose-lowering agents. One possibly explanation for this change in blood glucose is an improved diet and increased physical activity. However, this possiblity needs to be further examined via analyses of the changes in diet and exercise during FGM in the present study population (study remains ongoing). Previous studies showed that FGM improved blood glucose control better in patients with high HbA1c levels than those with relatively lower levels [14, 15]. Although these studies explained the positive correlation between baseline HbA1c and $\Delta \mathrm{A} 1 \mathrm{c}$, they did not calculate the EA1c in patients with high MG separately. Thus, we performed linear regressions among patients stratified according to $\mathrm{MG}$ to investigate different models of EA1c calculation in patients with different blood glucose control. Moreover, all of the variables we included in the models were from FGM; patients therefore did not need to provide additional blood samples or do other tests.

Kumagai et al. found that glucose values tended to be lower when measured using FGM than with CGM [16]. Moreover, it has been reported that the accuracy of data on the first day is lower than that on the other days during the FGM period $[17,18]$. As a result, total MG (MG over the 14-day FGM period) in FGM is lower than the actual measured blood glucose level, and the EA1c calculated with total MG only is also lower than the actual HbA1c. The accuracy is stable in the middle stage of FGM [18], and thus adjustment of the EA1c using MG on eighth day of FGM partially solves this problem. The correlation coefficient increased in the model that was adjusted with MG on the eighth day.

Dunn et al. found that EA1c was negatively correlated to scan rates [2]. Our result confirmed that finding. However, we found no correlation between scan rates and $\Delta \mathrm{A} 1 \mathrm{c}$, although we cannot exclude the influence of a smaller sample size and/or shorter study duration compared to the previous study.

This study has a number of limitations that need to be discussed. First, the change in diet and physical activity during FGM needs to be further studied. Secondly, findings using a larger sample size may be more convincing. Thirdly, our study was limited to a single center, which should be expanded. Moreover, although we did not allow any change in the dosage of glucose-lowering agents during FGM, we did permit changes in glucose-lowering agents immediately after the FGMs, in view of medical ethics; thus, the reduction in glucose level during the second FGM period may have been affected. However, the interval between the two FGMs (62 days) is sufficiently long to diminish the influence of hypoglycemic agent adjustment, and the influence of glucose-lowering agent adjustment on EA1c and HbA1c may be similar, so that it may have little influence on the relationship between EA1c and HbA1c in the second FGM. The correlations between EA1c and actual HbA1c in the first and second FGM period were similar in this study, which confirms our hypothesis.

In clinical work, the underestimation of actual HbA1c may result in a neglect of the effects of glucotoxicity during the past 3 months, which in turn may lead to diabetic complications. In studies, the use of EA1c may lead to a misjudgment of glycemic control in patients. We suggest a better model for EA1c calculation to resolve these problems. The proper use of EA1c will reduce both the number of blood samples taken and hospital visits. The results of this study may be useful for the application of FGM.

\section{CONCLUSIONS}

The EA1c in FreeStyle Libre is lower than the actual measured HbA1c. The improvement in glucose levels during FGM in these patients may contribute to the lowering of EA1c.

\section{ACKNOWLEDGEMENTS}

We thank the participants for their cooperation, and members of Endocrinology department of Nanjing First hospital for their support. 
Funding. This study was supported by the National Key R\&D Program of China (No. 2018YFC1314103), the National Natural Science Foundation of China (No. 81870563) and the Nanjing Municipal Science and Technology Project (No. 201803011). No Rapid Service Fee was received by the journal for the publication of this article.

Editorial Assistance. We thank Elliot Frank (retired) for providing English language assistance for this article. No funding was provided for this assistance.

Authorship. All named authors meet the International Committee of Medical Journal Editors (ICMJE) criteria for authorship for this article, take responsibility for the integrity of the work as a whole, and have given their approval for this version to be published.

Authorship Contributions. Design: Jianhua $\mathrm{Ma}$; Xiaofei Su; Conduct/data collection: Yun Shen; Rengna Yan; Huiying Wang; Bo Ding; Analysis: Yun Hu; Fengfei Li; Writing manuscript: Yun Hu.

Disclosures. Yun $\mathrm{Hu}$, Yun Shen, Rengna Yan, Fengfei Li, Bo Ding, Huiying Wang, Xiaofei $\mathrm{Su}$ and Jianhua Ma have nothing to disclose.

Compliance with Ethics Guidelines. The study protocol was approved by the Institutional Ethics Committee of Nanjing First Hospital, Nanjing Medical University (number KY20170904-04), and was in accordance with the Helsinki Declaration of 1964, as revised in 2013. All patient provided written informed consent forms to participate in the study.

Data Availability. The datasets generated during and/or analyzed during the current study are available from the corresponding author on reasonable request.

Open Access. This article is licensed under a Creative Commons Attribution-NonCommercial 4.0 International License, which permits any non-commercial use, sharing, adaptation, distribution and reproduction in any medium or format, as long as you give appropriate credit to the original author(s) and the source, provide a link to the Creative Commons licence, and indicate if changes were made. The images or other third party material in this article are included in the article's Creative Commons licence, unless indicated otherwise in a credit line to the material. If material is not included in the article's Creative Commons licence and your intended use is not permitted by statutory regulation or exceeds the permitted use, you will need to obtain permission directly from the copyright holder. To view a copy of this licence, visit http:// creativecommons.org/licenses/by-nc/4.0/.

\section{REFERENCES}

1. Al-Agha AE, Kafi SE, Zain Aldeen AM, Khadwardi RH. Flash glucose monitoring system may benefit children and adolescents with type 1 diabetes during fasting at Ramadan. Saudi Med J. 2017;38(4): 366-71.

2. Dunn TC, Xu Y, Hayter G, Ajjan RA. Real-world flash glucose monitoring patterns and associations between self-monitoring frequency and glycaemic measures: a European analysis of over 60 million glucose tests. Diabetes Res Clin Pract. 2018;137: 37-46.

3. Jangam S, Dunn T, Xu Y, Hayter G, Ajjan RA. Flash glucose monitoring improves glycemia in higher risk patients: a longitudinal, observational study under real-life settings. BMJ Open Diabetes Res Care. 2019;7(1):e000611.

4. Hellmund R, Weitgasser R, Blissett D. Cost calculation for a flash glucose monitoring system for adults with Type 2 diabetes mellitus using intensive insulin: a UK perspective. Eur Endocrinol. 2018;14(2): 86-92.

5. American Diabetes Association. 6. Glycemic targets: standards of medical care in diabetes-2019. Diabetes Care. 2019;42(Suppl 1):S61-S70.

6. Jovanovic L, Savas H, Mehta M, Trujillo A, Pettitt DJ. Frequent monitoring of A1C during pregnancy as a treatment tool to guide therapy. Diabetes Care. 2011;34(1):53-4.

7. Forbes A, Murrells T, Mulnier H, Sinclair AJ. Mean HbA 1c, HbA 1c variability, and mortality in people with diabetes aged 70 years and older: a 
retrospective cohort study. Lancet Diabetes Endocrinol. 2018;6(6):476-86.

8. Koenig RJ, Peterson CM, Jones RL, Saudek C, Lehrman M, Cerami A. Correlation of glucose regulation and hemoglobin A1c in diabetes mellitus. N Engl J Med. 1976;295(8):417-20.

9. Rohlfing CL, Wiedmeyer HM, Little RR, England JD, Tennill A, Goldstein DE. Defining the relationship between plasma glucose and $\mathrm{HbA}(1 \mathrm{c})$ : analysis of glucose profiles and $\mathrm{HbA}(1 \mathrm{c})$ in the Diabetes Control and Complications Trial. Diabetes Care. 2002;25(2):275-8.

10. Nathan DM, Turgeon H, Regan S. Relationship between glycated haemoglobin levels and mean glucose levels over time. Diabetologia. 2007;50(11): 2239-44.

11. Nathan DM, Kuenen J, Borg R, Zheng H, Schoenfeld $\mathrm{D}$, Heine RJ. Translating the A1C assay into estimated average glucose values. Diabetes Care. 2008;31(8):1473-8.

12. Haak T, Hanaire H, Ajjan R, Hermanns N, Riveline JP, Rayman G. Flash glucose-sensing technology as a replacement for blood glucose monitoring for the management of insulin-treated Type 2 diabetes: a multicenter open-label randomized controlled trial. Diabetes Ther. 2017;8(1):55-73.
13. Yaron M, Roitman E, Aharon-Hananel G, et al. Effect of flash glucose monitoring technology on glycemic control and treatment satisfaction in patients with type 2 diabetes. Diabetes Care. 2019;42(7):1178-84.

14. Dover AR, Stimson RH, Zammitt NN, Gibb FW. Flash glucose monitoring improves outcomes in a type 1 diabetes clinic. J Diabetes Sci Technol. 2017;11(2):442-3.

15. McKnight JA, Gibb FW. Flash glucose monitoring is associated with improved glycaemic control but use is largely limited to more affluent people in a UK diabetes centre. Diabetes Med. 2017;34(5):732.

16. Kumagai R, Muramatsu A, Fujii M, et al. Comparison of glucose monitoring between Freestyle Libre Pro and iP ro2 in patients with diabetes mellitus. J Diabetes Investig. 2019;10(3):851-6.

17. Leelarathna L, Wilmot EG. Flash forward: a review of flash glucose monitoring. Diabet Med. 2018;35(4):472-82.

18. Bailey T, Bode BW, Christiansen MP, Klaff LJ, Alva $\mathrm{S}$. The performance and usability of a factory-calibrated flash glucose monitoring system. Diabetes Technol Ther. 2015;17(11):787-94. 